\title{
Design Concepts of Polycarbonate-Based Intervertebral Lumbar Cages: Finite Element Analysis and Compression Testing
}

\author{
J. Obedt Figueroa-Cavazos, ${ }^{1}$ Eduardo Flores-Villalba, ${ }^{1}$ José A. Diaz-Elizondo, ${ }^{2}$ \\ Oscar Martínez-Romero, ${ }^{1}$ Ciro A. Rodríguez, ${ }^{1}$ and Héctor R. Siller ${ }^{1}$ \\ ${ }^{1}$ Tecnologico de Monterrey, Escuela de Ingeniería y Ciencias, 64849 Monterrey, NL, Mexico \\ ${ }^{2}$ Tecnologico de Monterrey, Escuela de Medicina, 64710 Monterrey, NL, Mexico
}

Correspondence should be addressed to Héctor R. Siller; hector.siller@itesm.mx

Received 16 November 2015; Revised 29 March 2016; Accepted 18 April 2016

Academic Editor: Tadeusz Mikołajczyk

Copyright (C) 2016 J. Obedt Figueroa-Cavazos et al. This is an open access article distributed under the Creative Commons Attribution License, which permits unrestricted use, distribution, and reproduction in any medium, provided the original work is properly cited.

\begin{abstract}
This work explores the viability of 3D printed intervertebral lumbar cages based on biocompatible polycarbonate (PC-ISO ${ }^{\circledR}$ material). Several design concepts are proposed for the generation of patient-specific intervertebral lumbar cages. The 3D printed material achieved compressive yield strength of $55 \mathrm{MPa}$ under a specific combination of manufacturing parameters. The literature recommends a reference load of $4,000 \mathrm{~N}$ for design of intervertebral lumbar cages. Under compression testing conditions, the proposed design concepts withstand between 7,500 and 10,000 N of load before showing yielding. Although some stress concentration regions were found during analysis, the overall viability of the proposed design concepts was validated.
\end{abstract}

\section{Introduction}

The combination of biotechnology and 3D printing has led to the rise of $3 \mathrm{D}$ bioprinting, which is a processing technique that promises to solve critical issues while finding printable biomaterials, increasing the capacity of precise positioning and including cell sources, in order to be successfully applied in diagnosis, personalized medicine, and regenerative medicine [1]. Literature that demonstrates the disruptiveness of this group of applications is spread out in several fields. Radenkovic et al. suggested the idea of manufacturing personalized human hollow organs with lower architectural complexity using detailed patient information, acquired by medical imaging, appropriate cell type, and 3D printing technology [2]. Other works showed the potential of medical and industrial applications of several classes of 3D printing techniques that may be useful for attending the future demand for organ transplants. For example, Yoo made a comparison among different 3D bioprinting technologies in order to evaluate their impact on human health and medical devices industry [3]. Visser et al. stated that the potential application of 3D bioprinting in medicine will evolve into tissue printing in the near future [4].
3D printing of implants, prosthesis, and other medical devices can be considered an important stage of the full development of 3D bioprinting applications. Particularly, design of prosthesis and implants is nowadays embracing the use of 3D printing technologies as FDM (Fused Deposition Modeling), in order to solve the need for customization and the need for providing a fast response in surgical interventions [5]. Some previous works show that it is possible to satisfy the main features required in customized medical devices such as strength, sterility, dimensional stability, and safety. For example, Rankin et al. used a FDM machine for surgical retractors prototyping. This prototype was sterilized and tolerated the tangential force needed to fulfill the requirements before failure, both before and after exposure to sterilization [6].

A specific case of the need of customized implants is column surgery. This is performed in order to ease pathologies associated with back pain that are sometimes caused by deterioration of surrounding fibrous ring of intervertebral discs, resulting in spinal disc herniation. The column surgery that deals with this illness is usually known as spinal fusion surgery, where the vertebrae gradually fuse into a single body with the introduction of an intervertebral cage implant. Spinal fusion is done most commonly in the lumbar region 




Figure 1: Representation of the lumbar cage implants (vertebrae images courtesy of Centro de Tecnologia da Informação Renato Archer).

of the spine, but it is also used to treat cervical and thoracic regions. The main function of an intervertebral cage implant is to fill the intervertebral space in order to facilitate the process of osseointegration and to provide mechanical support through an optimal load distribution and an interbody fusion balance fixation.

Figure 1 shows the conceptual representation of an intervertebral cage implant system. Typically, intervertebral cage implants are manufactured with medical grade titanium alloys (Ti6Al4V), polyether ether ketone (PEEK), and composite materials. But now, with the appearance of $3 \mathrm{D}$ printing and Additive Manufacturing Technologies like Fusion Deposition Modeling (FDM), the potential for developing customer oriented and reliable cage implants is higher. The following sections of this work explain the development of a process chain for taking advantage of FDM in column surgery implants customization.

\section{Related Work}

There are a limited number of research studies that explore the design and rapid manufacturing of patient-specific implants for column surgery. de Beer and van der Merwe developed a study of the rapid manufacturing of metallic implants. They used computed tomography (CT), direct laser metal sintering (DMLS), and mechanical testing in order to propose a process chain for customization of intervertebral disc implants [7]. In the same matter, Domanski et al. used three Additive Manufacturing Technologies: Fused Deposition Modeling, Powder Based 3D Printing (3DP), and Selective Laser Melting (SLM). They performed Finite Element Analysis for strength validation and they compared surface roughness of each prototype developed [8]. Espalin et al. suggested the use of FDM for the freeform manufacturing of several types of implants. They concentrated their research approach on cranial reconstruction, but they suggested this technique for the customization of orthopedic spacers [9]. Chougule et al. used noninvasive imaging, reverse engineering, and FDM for the development of specific implants for minimum invasive spine surgeries. They suggested that this process chain could provide valuable medical information and powerful diagnostic tool for surgeons to understand the complex internal anatomy of the patient [10]. A work that precedes the one presented here explores the use of FDM and common 3D printing machinery, in the development of design concepts of polymeric spinal surgery implants [11]. It was an exploratory study that evaluated the feasibility of implant customization with this kind of techniques and lays the foundations of the present work.

\section{Objective}

There is a clear need to evaluate the viability of the use of $3 \mathrm{D}$ printing for the customization of column surgery implants. Thus, the main objective of this research is to validate a series of design concepts of a specific case of column implants (called "intervertebral lumbar cages") with the aid of FDM, computational simulations, and experimental testing. Furthermore, this work is intended to lay the basis for the implementation of these medical devices in orthopedic surgeries.

\section{Materials and Methods}

This section covers the set-up of a proposed process chain for lumbar cage material selection, design, prototyping through FDM, mechanical testing, and Finite Element Analysis.

4.1. Materials. The proposed polymer for this research is PCISO (polycarbonate-ISO), an industrial thermoplastic that can be sterilized by several methods like ethylene oxide and gamma radiation, according to the work of Perez et al. In this work sterility testing was performed with successful results for material deposited with FDM process [12]. Cunha et al. evaluated safety and radiation attenuation properties of PCISO material and established a method for its clinical use in customized Gynecological Brachytherapy Applicators. The work concluded that PC-ISO is a suitable material for this application in a clinical setting [13].

In the case of the material biocompatibility, there are several studies ordered by the material supplier that confirm that the material is not toxic, does not present allergenic potential, and does not have irritant effects. Among the studies mentioned above there is, for example, the ISO Acute Systemic Injection Test, which was designed for screening PC-ISO extracts for potential toxic effects as a result of a single-dose systemic injection in mice. The study confirmed that the animals did not present signs of toxicity in comparison with the control. Regarding mechanical properties of the chosen material, it has a specified ultimate tensile strength of $57 \mathrm{MPa}$ and a modulus of elasticity of $2 \mathrm{GPa}$, properties that made this polymer competitive with other engineered materials for implants [11].

4.2. Prototypes. The implant design for FDM took into account several design guidelines for the appropriate deposition of the polymeric material, in order to improve its mechanical performance during tensile or compressive testing and for increasing the dimensional and geometrical accuracy. Some of these design guidelines were formulated by Ahn et al., based on the results of extensive experimentation [14]. Derived from that work, the following guidelines are 


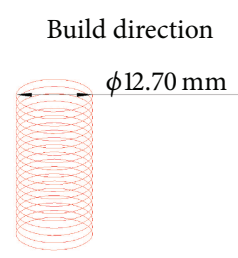

Part interior style



(a)

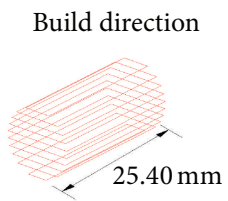

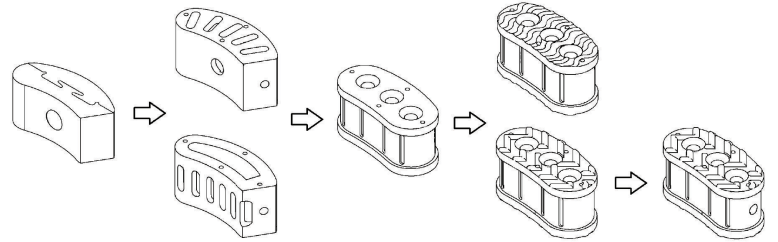

FIGURE 3: Evolution of lumbar cage design concepts.

TABLE 2: Fused Deposition Modeling process specifications.

\begin{tabular}{lll}
\hline Nozzle diameter & $0.30 \mathrm{~mm}$ & $0.40 \mathrm{~mm}$ \\
\hline Slice height & $0.17 \mathrm{~mm}$ & $0.25 \mathrm{~mm}$ \\
Contour width & $0.35 \mathrm{~mm}$ & $0.50 \mathrm{~mm}$ \\
Part raster width & $0.35 \mathrm{~mm}$ & $0.50 \mathrm{~mm}$ \\
Visible surface raster & $0.30 \mathrm{~mm}$ & $0.43 \mathrm{~mm}$ \\
Internal raster & $0.40 \mathrm{~mm}$ & $0.61 \mathrm{~mm}$ \\
\hline
\end{tabular}

FIGURE 2: Build direction and part interior style of cylindrical test specimens: (a) transverse-vertical and (b) horizontal-axial configurations.

TABLE 1: System specifications of FDM machine.

\begin{tabular}{lc}
\hline Configuration & \\
\hline Build envelope $(X Y Z)$ & $406 \times 355 \times 406 \mathrm{~mm}$ \\
\hline Material delivery & Two build material canisters $1508 \mathrm{cc}$ \\
& $\begin{array}{c}\text { Two support material canisters } 1508 \mathrm{cc} \\
\text { Autochangeover between canisters }\end{array}$ \\
\hline Material options & PC-ISO \\
\hline & $0.330 \mathrm{~mm}$ \\
Layer thickness & $0.254 \mathrm{~mm}$ \\
& $0.178 \mathrm{~mm}$ \\
\hline Support structure & Breakaway Support System $\left(\mathrm{BASS}^{\mathrm{TM}}\right)$ \\
\hline
\end{tabular}

used in this particular case study:

(i) A negative air gap, meaning that two FDM layers partially occupy the same space, will increase both strength and stiffness.

(ii) The build orientation could improve the part accuracy and strength.

Prior to the exploration of patient-specific lumbar cage process chain, cylindrical specimens with two different configurations of build directions were subject to compression strength testing (Figure 2). This procedure was carried out as screening experimentation in order to establish the material mechanical properties for a Finite Element Analysis of the lumbar cage concepts. For the manufacture of the cylindrical specimens and implant design concepts, a FDM machine Stratasys Fortus $400 \mathrm{mc}$ was used and two different machine nozzles were configured for the deposition process (Tables 1 and 2). According to the supplier of PC-ISO material, the specific gravity is 1.20 , which is equivalent to $0.0012 \mathrm{~g} / \mathrm{mm}^{3}$ in density [15]. Taking as reference this value and the final weights of the cylindrical specimens, the fill percentage obtained with $0.30 \mathrm{~mm}$ nozzle is approximately $88 \%$ and the fill percentage obtained with the $0.40 \mathrm{~mm}$ nozzle is approximately $92 \%$, which may influence the strength behavior.

The design criteria for lumbar cage geometries were established by the analysis of patents and commercial products, in which the predominant factor found was the load distribution for providing minimum damage to vertebrae [16-19].

It is important to point out that the mechanical response to fatigue testing must be considered in further research since there is evidence in the literature that repetitive loading can cause vulnerability to column mechanical damage [20], and there is limited fatigue data in the literature for FDM deposited materials [21].

The evolution of all design attempts is shown in Figure 3, in which the geometries presented in previous research are also exhibited [11].

In this work, four different lumbar cage design concepts are proposed, as an optimization alternative in the search for appropriate 3D printed implants. These lumbar cage design concepts were tested in a similar set-up to that used for cylindrical specimens. Solid models of the proposed lumbar cages were drawn using a generic CAD tool. The dimensions ranges of the prototypes were about $13.4 \mathrm{~mm}$ in width and $28.7 \mathrm{~mm}$ in length, having a height of 13 millimeters. Also, all the designs included geometrical features that facilitate osseointegration. It is important to point out that the design concepts include antiskid systems required for appropriate implant fixation, but these geometrical features could generate stress concentration. Figure 4 presents the proposed lumbar cage design: (a) a flat lumbar cage that does not have an antiskid system; (b) a lumbar cage with an antiskid system with undulating features; (c) a lumbar cage with antiskid system with triangular features; and (d) the same geometry that proposed lumbar cage design (c), but with a handling hole in the side view (the handling hole is used to hold the lumbar cage during implantation).

4.3. Compression Strength Testing. Compressive strength tests for each cylindrical specimen and each lumbar cage design concept were conducted using a Shimadzu Universal 


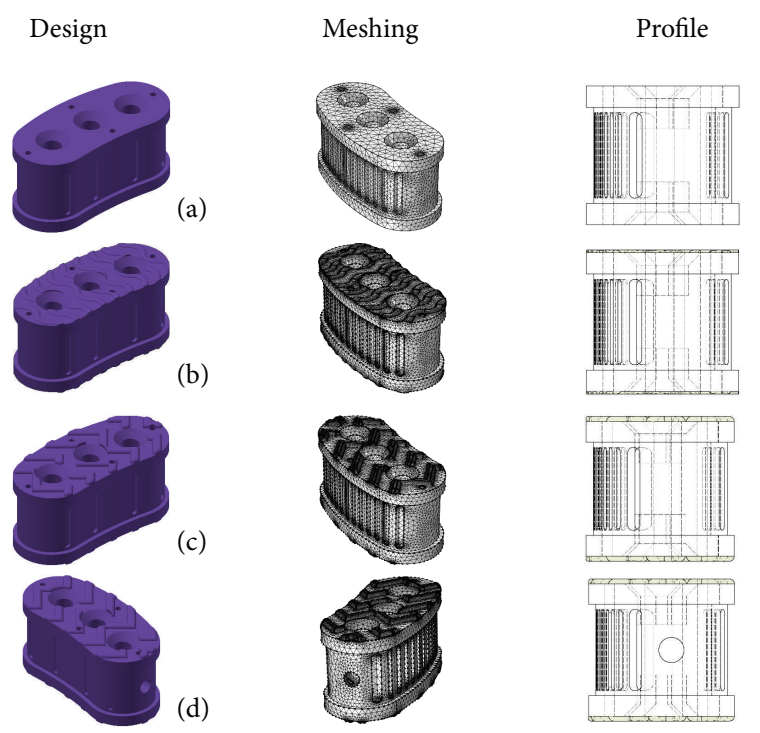

FIgURE 4: Proposed lumbar cage new designs.

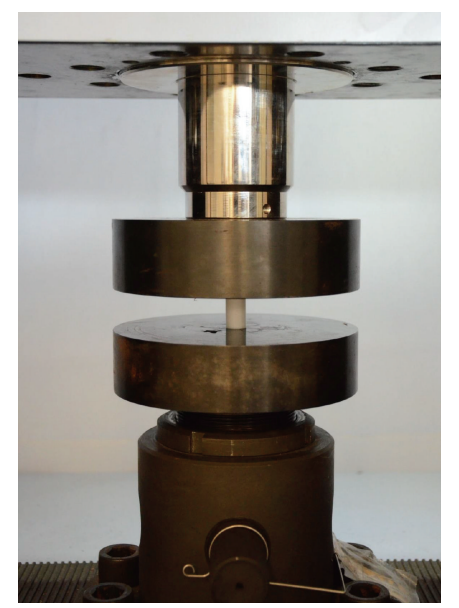

FIGURE 5: Test system equipped with a $25 \mathrm{kN}$ load cell.

Testing Machine equipped with a $25 \mathrm{kN}$ load cell. The tests performed on cylindrical specimens were based on the "ASTM.D695.2010, Standard Test Method for Compressive Properties of Rigid Plastics" [22], using $6 \mathrm{~mm} / \mathrm{min}$ as crosshead speed (Figure 5).

4.4. Simulation Settings. The Finite Element Analysis (FEA) was performed with the aid of COMSOL Multiphysics 4.3 software, to automatically generate tetrahedral elements and for performing the computational simulation. It is a common practice to use automatic tetrahedral mesh generators to discretize complex 3D structural components. This type of mesh generators can handle complex geometries with a minimum of human intervention (as compared to, e.g., the manual generation of a mesh of hexahedral elements). The solver used to perform the 3D calculations was embedded in the software. Mesh size was predefined in a range of 0.286 and $2.290 \mathrm{~mm}$ with a maximum element growth rate

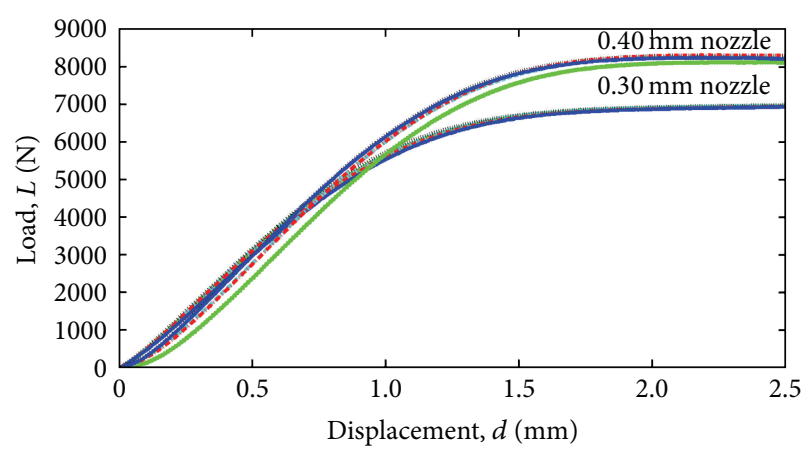

FIGURE 6: Comparison of the compression test of PC-ISO cylindrical test specimens, printed with $0.30 \mathrm{~mm}$ and 0.40 nozzle configuration.

TABle 3: Mechanical properties of PC-ISO cylindrical test specimens.

\begin{tabular}{lccc}
\hline Mechanical properties & Unit & $0.30 \mathrm{~mm}$ & $0.40 \mathrm{~mm}$ \\
\hline Compressive yield strength & $\mathrm{MPa}$ & 40 & 55 \\
Young's modulus & $\mathrm{GPa}$ & 1.2220 & 1.2580 \\
Poisson's ratio & 1 & 0.3928 & 0.4287 \\
Density & $\mathrm{Kg} / \mathrm{m}^{3}$ & 1,060 & 1,110 \\
\hline
\end{tabular}

of 1.45. Mechanical properties of the proposed lumbar cages were assumed to be homogeneous and linear elastic. The mechanical properties of Table 3 were taken as simulation references for each proposed prototype.

4.5. Reference Column Load. In order to establish a good approximation of the load that must be used for simulating average column loads, an additional literature review was made. Wilke et al. estimated an interdiscal pressure of 1.8 MPa, equivalent of $3,240 \mathrm{~N}$ in a disc area of $1800 \mathrm{~mm}^{2}$, in the L4-L5 disc while an average person was holding a $20 \mathrm{~kg}$ object $600 \mathrm{~mm}$ away from the chest [23]. Schultz et al. estimated that the interdiscal pressure varies depending on the body position and type of activity, taking values from $0.270 \mathrm{MPa}\left(486 \mathrm{~N}\right.$ of load over $1800 \mathrm{~mm}^{2}$ ) in a relaxed standing position to $1.62 \mathrm{MPa}\left(2,916 \mathrm{~N}\right.$ of load over $\left.1800 \mathrm{~mm}^{2}\right)$ in the most strenuous task examined [24]. Therefore, considering the average loads from the literature, a 4,000 N axial force was taken as reference load and the bottom face of the prototype was rigidly fixed for motion constraint.

\section{Results and Discussion}

5.1. Compression Strength Testing. The results of this screening experimentation for horizontal-axial build configuration were discarded since they presented lower strength behavior $(7,800 \mathrm{~N})$ in comparison to those with transverse-vertical build configuration for similar conditions (8,300 N). Figure 6 shows results of the compressive strength test of PC-ISO specimens manufactured by $0.30 \mathrm{~mm}$ nozzle in transversevertical build direction and $0.40 \mathrm{~mm}$ nozzle in transversevertical build direction. In order to evaluate the repeatability of the FDM process, several replications were conducted. The 


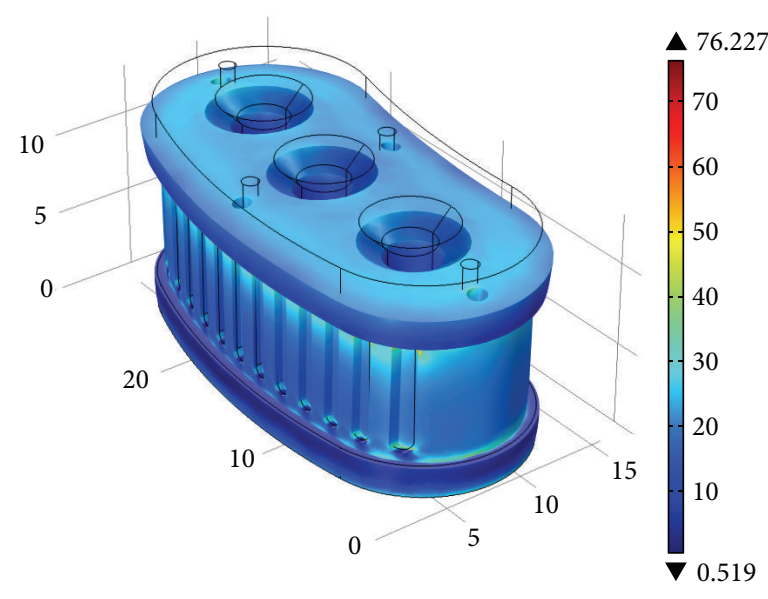

(a) Max 76.227 MPa

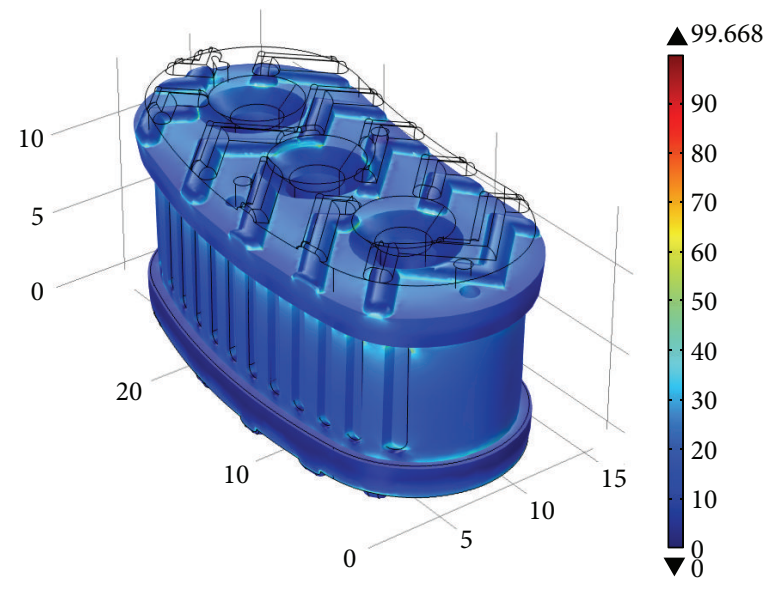

(c) $\operatorname{Max} 99.668 \mathrm{MPa}$

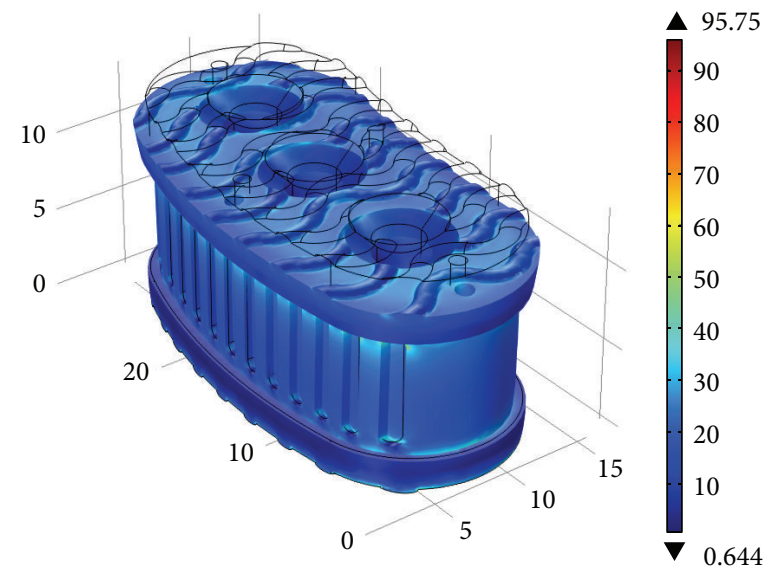

(b) Max $95.750 \mathrm{MPa}$



(d) Max 133.680 MPa

FIGURE 7: Volumetric analysis: von Mises stress distribution with 4,000 N as reference load.

average of these loads was of $7,776 \mathrm{~N}$ and the standard deviation was of $102.3 \mathrm{~N}$. Therefore, the process has a deviation error of $1.32 \%$ between the five experimental trials, a number that indicates a good repeatability of the process.

Table 3 shows the mechanical properties of PC-ISO cylindrical test specimens obtained from the initial mechanical compressive testing, in order to be used as preliminary data for the Finite Element Analysis. Compressive yield strength was calculated by dividing the load carried by the specimen at the yield point by the original minimum cross-sectional area of the specimen $\left(126.7 \mathrm{~mm}^{2}\right)$. Figure 6 shows the comparison of the compression test of PC-ISO cylindrical test specimens of both $0.30 \mathrm{~mm}$ and $0.40 \mathrm{~mm}$ nozzle configurations.

5.2. Simulation Results for Lumbar Cage Concepts. Simulations results are explained in terms of the average von Mises stresses exhibited in different cross sections of the four design concepts. Figure 7 shows the Finite Element Analysis of prototypes manufactured with the $0.40 \mathrm{~mm}$ nozzle in a full isometric view, with a top cross-sectional area of $257 \mathrm{~mm}^{2}$. Using the $4,000 \mathrm{~N}$ force as reference load, it is shown that the average von Mises stresses are up to $30 \mathrm{MPa}$ for all prototypes.
Complex geometry shows higher maximum von Mises stress. Figure 8 shows the middle cross section with an area of $268 \mathrm{~mm}^{2}$ for concepts (a), (b), and (c). Concept (d) has a middle cross section area of $262 \mathrm{~mm}^{2}$. As expected, all design concepts exhibited average von Mises stress of approximately $15 \mathrm{MPa}$. Stress increases in the outer regions of the cross section due to the complex geometry of the outside surface of the implant. In the case of concept (d), the maximum stress is higher compared to the other concepts due to the stress concentration effect of the handling hole.

Figure 9 shows the cross section of the top plane where the volumetric analysis (from Figure 7) shows the maximum von Mises stress. In this plane, the cross-sectional area is $247 \mathrm{~mm}^{2}$ due to the blind holes incorporated in the design concept. Average von Misses stress should be 16.2 MPa. Stress concentration regions produce localized values up to $78.8 \mathrm{MPa}$.

5.3. Experimental Results for Lumbar Cage Concepts. Figure 10 shows a comparison between compressive strength tests of the proposed lumbar cage design (a) manufactured by the $0.30 \mathrm{~mm}$ nozzle and $0.40 \mathrm{~mm}$ nozzle. It is clearly 


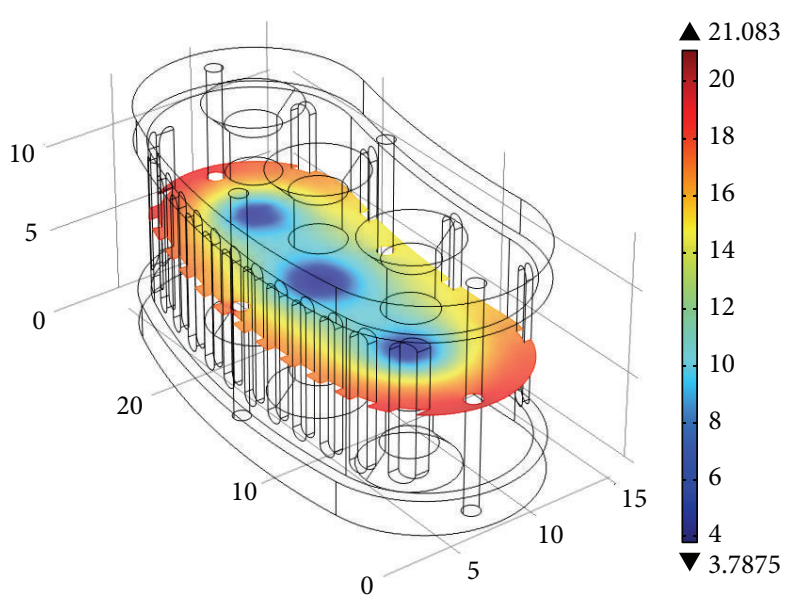

(a) Max $21.083 \mathrm{MPa}$

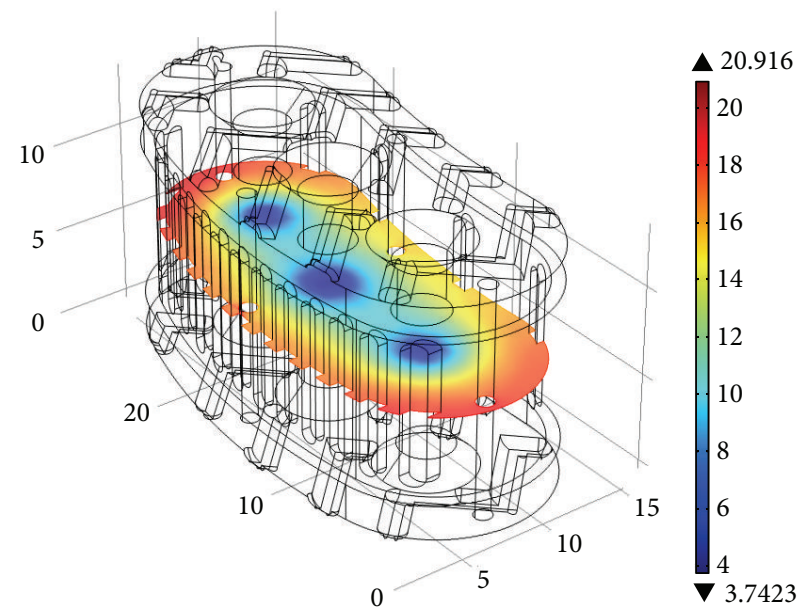

(c) $\operatorname{Max} 20.916 \mathrm{MPa}$

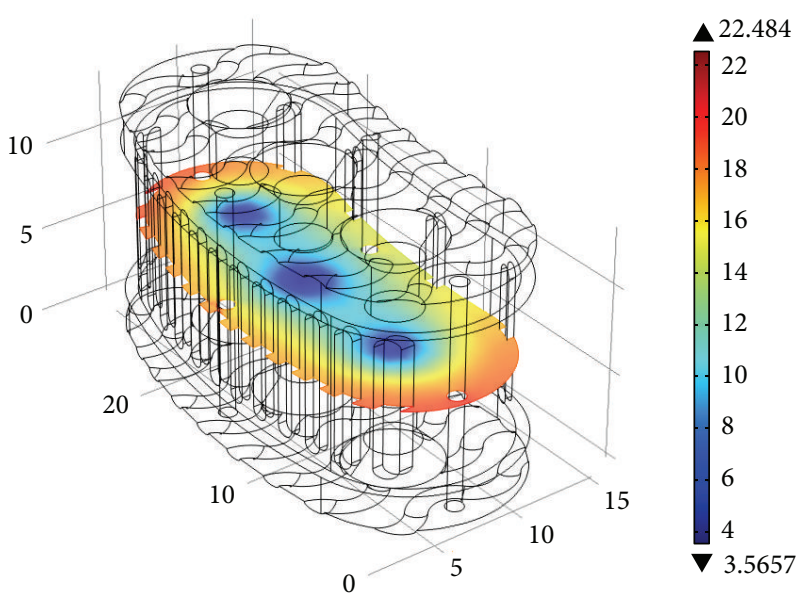

(b) Max 22.484 MPa



(d) $\operatorname{Max} 58.115 \mathrm{MPa}$

FIGURE 8: Cross section analysis for middle plane: von Mises stress distribution with 4,000 N as reference load.

seen from the same figure that the lumbar cage design manufactured by the $0.40 \mathrm{~mm}$ nozzle has better mechanical properties than those manufactured by the $0.30 \mathrm{~mm}$ nozzle. Further experimentation was limited to the $0.40 \mathrm{~mm}$ nozzle configuration.

Figure 11 shows the measured load $(L)$ versus displacement $(d)$ behavior of prototypes manufactured with the $0.40 \mathrm{~mm}$ nozzle. The best mechanical behavior is found in those design concepts with simpler geometrical elements, such as design (a) with the flat surface and design (b) with undulating features. However, the design concepts with more complex geometry (such as (c) and (d)) facilitate osseointegration and the surgical procedure during implantation.

5.4. Discussion. Based on the cylindrical samples, the reference compressive yield strength to consider is $55 \mathrm{MPa}$. The middle plane simulation analysis shows that, on average, stress level is well below the compressive yield strength of the FDM-printed PC-ISO material. Only in the case of concept (d), the maximum stress level exceeds the limit by small percentage (6\%).
From the compression testing experimental results of the cylindrical specimens and the design concepts (Figures 6 and 10 ), the mechanical behavior exhibited at $1 \mathrm{~mm}$ of displacement is similar in terms of engineering stress. Specifically, the cylindrical specimens exhibited an approximate engineering stress of $54 \mathrm{Mpa}$ (estimated with a cross section of $126 \mathrm{~mm}^{2}$ and $6800 \mathrm{~N}$ ) while the engineering stress at the middle cross section (estimated with $268 \mathrm{~mm}^{2}$ and 15,000 N) of the design concepts is approximately $55 \mathrm{Mpa}$.

Local maximum stress levels are above the material strength in some regions. For the top plane analysis, all regions with maximum stress are above the material strength (between 14 and 43\% higher stress compared to the material strength). The volumetric analysis shows regions that exceed the material strength by a large percentage (up to $143 \%$ in the case of design concept (d)).

In contrast to the FEM analysis results, using $4,000 \mathrm{~N}$ as the reference load, the actual compression test shows that the various design concepts are robust. For the case of design concepts (a) and (b), the yield behavior starts at approximately 


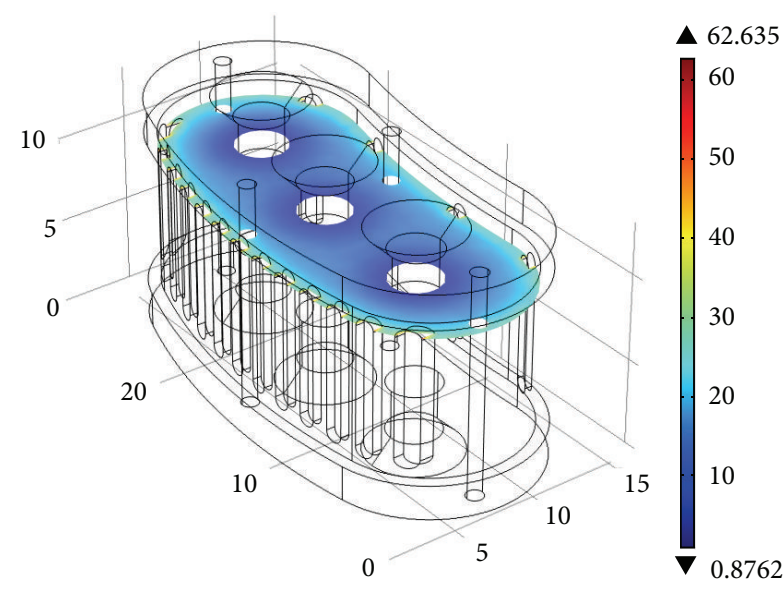

(a) Max 62.635 MPa

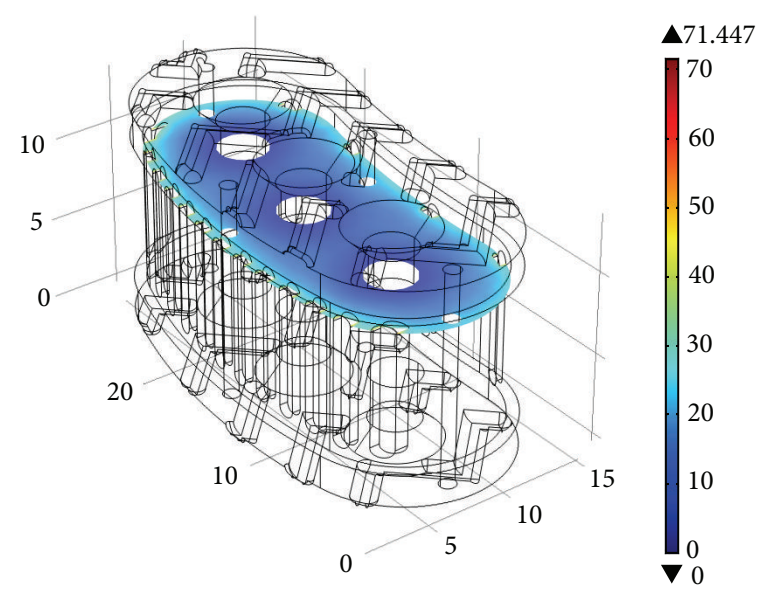

(c) Max $71.447 \mathrm{MPa}$

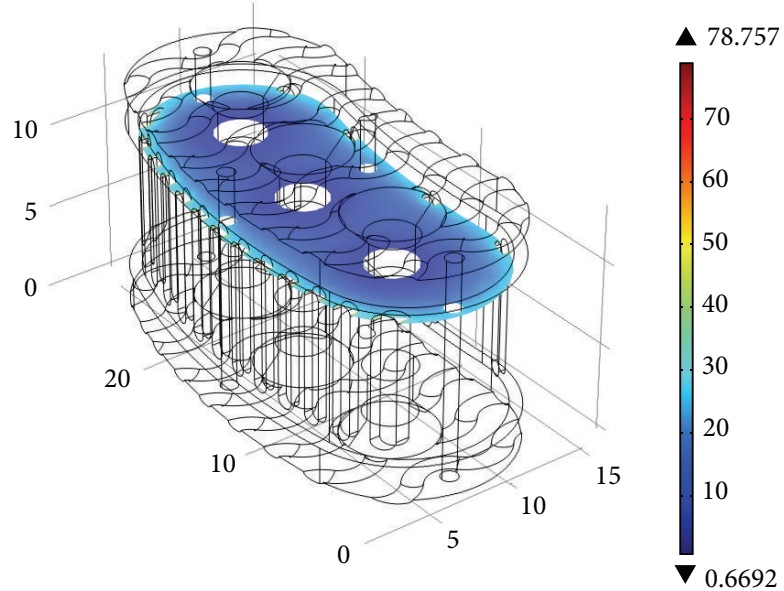

(b) Max $78.757 \mathrm{MPa}$

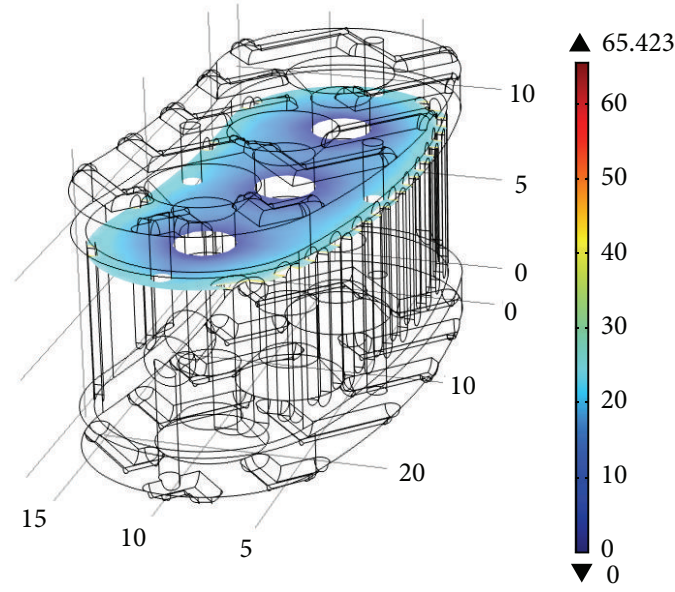

(d) Max 65.423 MPa

FIGURE 9: Cross section analysis for top plane: von Mises stress distribution with 4,000 $\mathrm{N}$ as reference load.

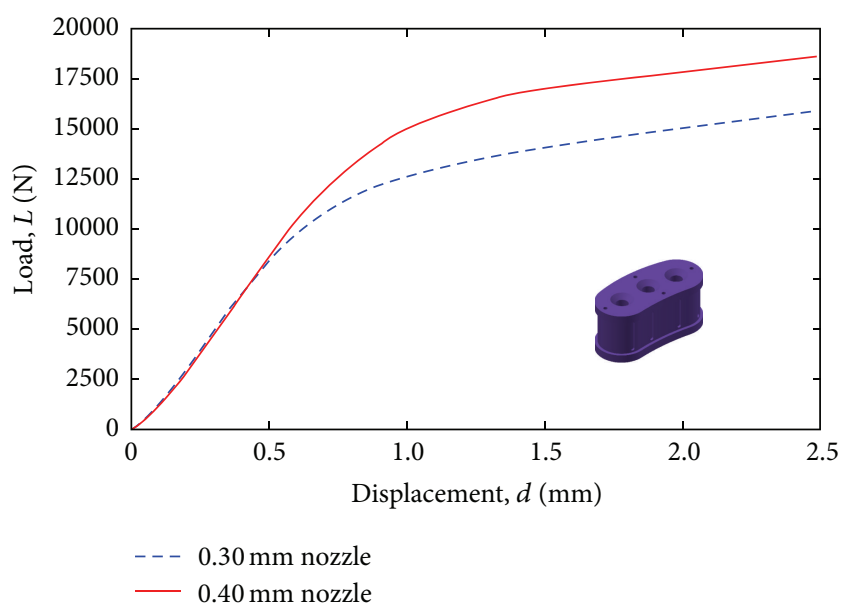

FIGURE 10: Compression testing for design concept (a), with different FDM nozzle configurations ( 0.30 versus $0.40 \mathrm{~mm}$ ).

$10,000 \mathrm{~N}$. A load of approximately $7,500 \mathrm{~N}$ is supported by design concepts (c) and (d) before yielding is observed.
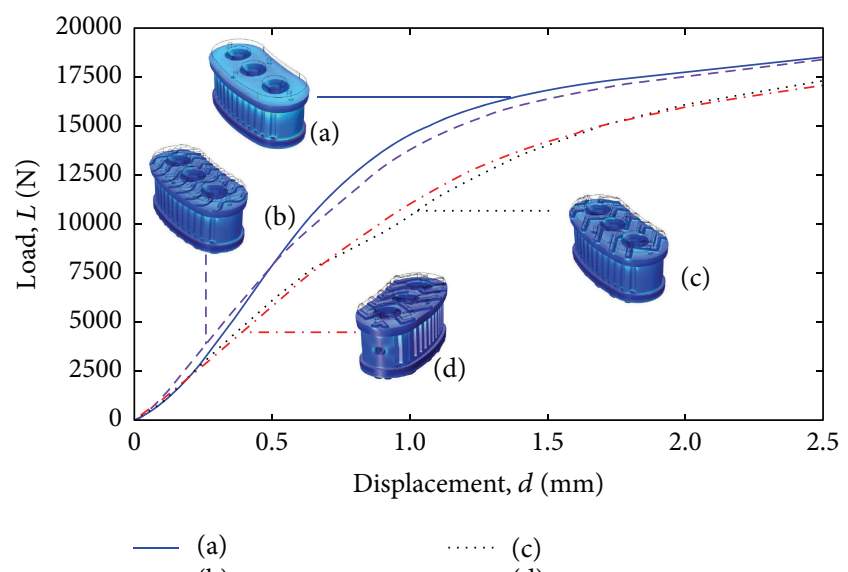

(b) $\quad \cdot \cdots$ (d)

FIGURE 11: Compression testing for all design concepts with $0.40 \mathrm{~mm}$ nozzle configuration.

The FDM process cannot produce sharp corners due to geometry of the filament used for generation of each layer. 
Therefore, this additive manufacturing process is beneficial to avoid stress concentration regions in the printed intervertebral lumbar cages. In addition, the FEM analysis shows that the stress concentration areas are quite localized. Therefore, it can be concluded that the high loads supported by the different design concepts are due to a combination of (a) localized stress concentration regions, (b) additive manufacturing process that intrinsically reduces stress concentration geometries, and (c) ductile nature of the PC-ISO material.

In order to further validate the proposed design concepts, additional testing under dynamic conditions is needed in order to assess the fatigue response of the material. In terms of the design concepts, additional refinements are required in order to reduce the amount of stress concentration due to sharp changes in geometry.

\section{Conclusions and Future Work}

This work has shown the viability of FDM-printed intervertebral lumbar cages based on biocompatible polycarbonate (PC-ISO material). Several design concepts are proposed for the generation of patient-specific intervertebral lumbar cages. Finite Element Analysis and compression testing show the viability of the proposed design concepts. The part interior style has a more significant influence than the build direction of the material deposition. Furthermore, PC-ISO material showed a high repeatability in the manufacture process for transverse-vertical build direction and solid part interior style parameters, achieving compressive yield strength of $55 \mathrm{MPa}$. The literature recommends a reference load of $4,000 \mathrm{~N}$ for design of intervertebral lumbar cages. Under compression testing conditions, the FDM-printed intervertebral lumbar cages withstand between 7,500 and 10,000 $\mathrm{N}$ of load before showing yielding.

Further research must be carried out with in vitro and in vivo testing in order to guarantee the full viability of the intervertebral lumbar cage implants that have been proposed in this work. Specifically, the validation should be carried out through the analysis of biocompatibility and osseointegration with the surrounding tissue, towards the full integration of $3 \mathrm{D}$ printed implants in the medical practice. The benefits of using $3 \mathrm{D}$ printing for each specific patient should potentially increase the ergonomics, simplify the procedure, and bring overall better personalized results once validated.

\section{Competing Interests}

The authors declare that there are no competing interests regarding the publication of this paper.

\section{Acknowledgments}

The authors would like to acknowledge the support of Tecnológico de Monterrey through its Research Group in Advanced Manufacturing. Support was also provided from the MADiT "National Lab of Additive Manufacturing, 3D Digitizing and Computerized Tomography" at Universidad Nacional Autónoma de México and from Centro de Tecnologia da Informação Renato Archer. Finally, the authors of this work would like to express their gratitude to Jan Lammel Lindemann, for his valuable comments.

\section{References}

[1] G. Gao and X. Cui, "Three-dimensional bioprinting in tissue engineering and regenerative medicine," Biotechnology Letters, vol. 38, no. 2, pp. 203-211, 2016.

[2] D. Radenkovic, A. Solouk, and A. Seifalian, "Personalized development of human organs using 3D printing technology," Medical Hypotheses, vol. 87, pp. 30-33, 2016.

[3] S.-S. Yoo, "3D-printed biological organs: medical potential and patenting opportunity," Expert Opinion on Therapeutic Patents, vol. 25, no. 5, pp. 507-511, 2015.

[4] J. Visser, F. P. Melchels, W. J. Dhert, and J. Malda, "Tissue printing; the potential application of 3D printing in medicine," Nederlands Tijdschrift voor Geneeskunde, vol. 157, no. 52, Article ID A7043, 2013.

[5] V. Petrovic, J. V. Haro Gonzalez, O. Jordá Ferrando, J. Delgado Gordillo, J. R. Blasco Puchades, and L. Portoles Grinan, "Additive layered manufacturing: sectors of industrial application shown through case studies," International Journal of Production Research, vol. 49, no. 4, pp. 1061-1079, 2011.

[6] T. M. Rankin, N. A. Giovinco, D. J. Cucher, G. Watts, B. Hurwitz, and D. G. Armstrong, "Three-dimensional printing surgical instruments: are we there yet?" Journal of Surgical Research, vol. 189, no. 2, pp. 193-197, 2014.

[7] N. de Beer and A. van der Merwe, "Patient-specific intervertebral disc implants using rapid manufacturing technology," Rapid Prototyping Journal, vol. 19, no. 2, pp. 126-139, 2013.

[8] J. Domanski, K. Skalski, R. Grygoruk, and A. Mróz, "Rapid prototyping in the intervertebral implant design process," Rapid Prototyping Journal, vol. 21, no. 6, pp. 735-746, 2015.

[9] D. Espalin, K. Arcaute, D. Rodriguez, F. Medina, M. Posner, and R. Wicker, "Fused deposition modeling of patient-specific polymethylmethacrylate implants," Rapid Prototyping Journal, vol. 16, no. 3, pp. 164-173, 2010.

[10] V. N. Chougule, A. V. Mulay, and B. B. Ahuja, "Development of patient specific implants for minimum invasive spine surgeries (MISS) from non-invasive imaging techniques by reverse engineering and additive manufacturing techniques," Procedia Engineering, vol. 97, pp. 212-219, 2014.

[11] O. Figueroa, C. A. Rodríguez, H. R. Siller et al., "Lumbar cage design concepts based on additive manufacturing," in High Value Manufacturing: Advanced Research in Virtual and Rapid Prototyping, P. H. da Silva Bártolo, A. C. S. de Lemos, A. M. H. Pereira et al., Eds., chapter 102, CRC Press, New York, NY, USA, 2013.

[12] M. Perez, M. Block, D. Espalin et al., "Sterilization of FDMmanufactured parts," in Proceedings of the 23rd Annual International Solid Freeform Fabrication Symposium (SFF '12), pp. 285296, Austin, Tex, USA, August 2012.

[13] J. Cunha, R. Sethi, K. Mellis et al., "WE-F-16A-01: commissioning and clinical use of PC-ISO for customized, 3D printed, gynecological brachytherapy applicators," Medical Physics, vol. 41, no. 6, article 514, 2014.

[14] S.-H. Ahn, M. Montero, D. Odell, S. Roundy, and P. K. Wright, "Anisotropic material properties of fused deposition modeling ABS," Rapid Prototyping Journal, vol. 8, no. 4, pp. 248-257, 2002.

[15] Stratasys, Production-Grade Thermoplastic for Fortus 3D Production Systems, Stratasys, Eden Prairie, Minn, USA, 2015. 
[16] B. Schafer and H. Halm, "Intervertebral implant," United States of America Patent US 6,143,032, 2000.

[17] S. A. Webb, A. P. Moreno, M. E. Mitchell, and A. C. Smith, "Spinal implant," United States of America Patent US 7,806,932 B2, 2010.

[18] P. P. Varga and J. W. Ogilvie, "Intervertebral spacer," United States of America Patent US 6,579,318 B2, 2003.

[19] F. K. Fuss and R. J. Sabitzer, "Implant for insertion between spinal column vertebrae," United States of America Patent US 6,562,072 B1, 2003.

[20] M. A. Adams and P. Dolan, "Spine biomechanics," Journal of Biomechanics, vol. 38, no. 10, pp. 1972-1983, 2005.

[21] J. Lee and A. Huang, "Fatigue analysis of FDM materials," Rapid Prototyping Journal, vol. 19, no. 4, pp. 291-299, 2013.

[22] ASTM D695-10, Standard Test Method for Compressive Properties of Rigid Plastics, ASTM International, West Conshohocken, Pa, USA, 2010, https://www.astm.org/.

[23] H.-J. Wilke, P. Neef, B. Hinz, H. Seidel, and L. Claes, "Intradiscal pressure together with anthropometric data-a data set for the validation of models," Clinical Biomechanics, vol. 16, supplement 1, pp. S111-S126, 2001.

[24] A. Schultz, G. Andersson, R. Ortengren, K. Haderspeck, and A. Nachemson, "Loads on the lumbar spine. Validation of a biomechanical analysis by measurements of intradiscal pressures and myoelectric signals," The Journal of Bone \& Joint Surgery-American Volume, vol. 64, no. 5, pp. 713-720, 1982. 


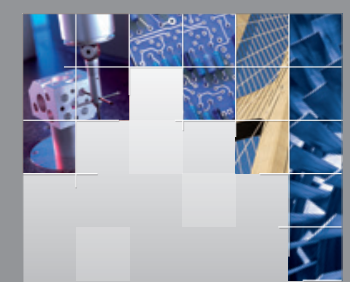

\section{Enfincering}


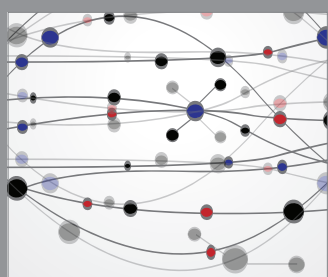

The Scientific World Journal

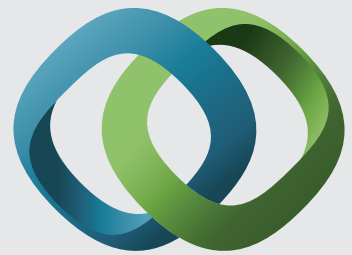

\section{Hindawi}

Submit your manuscripts at

http://www.hindawi.com
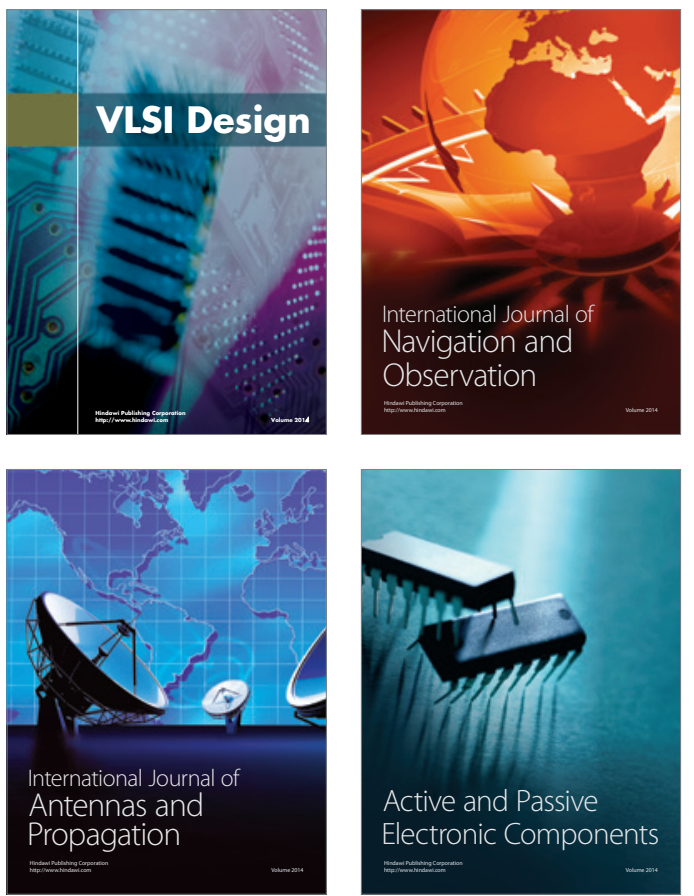
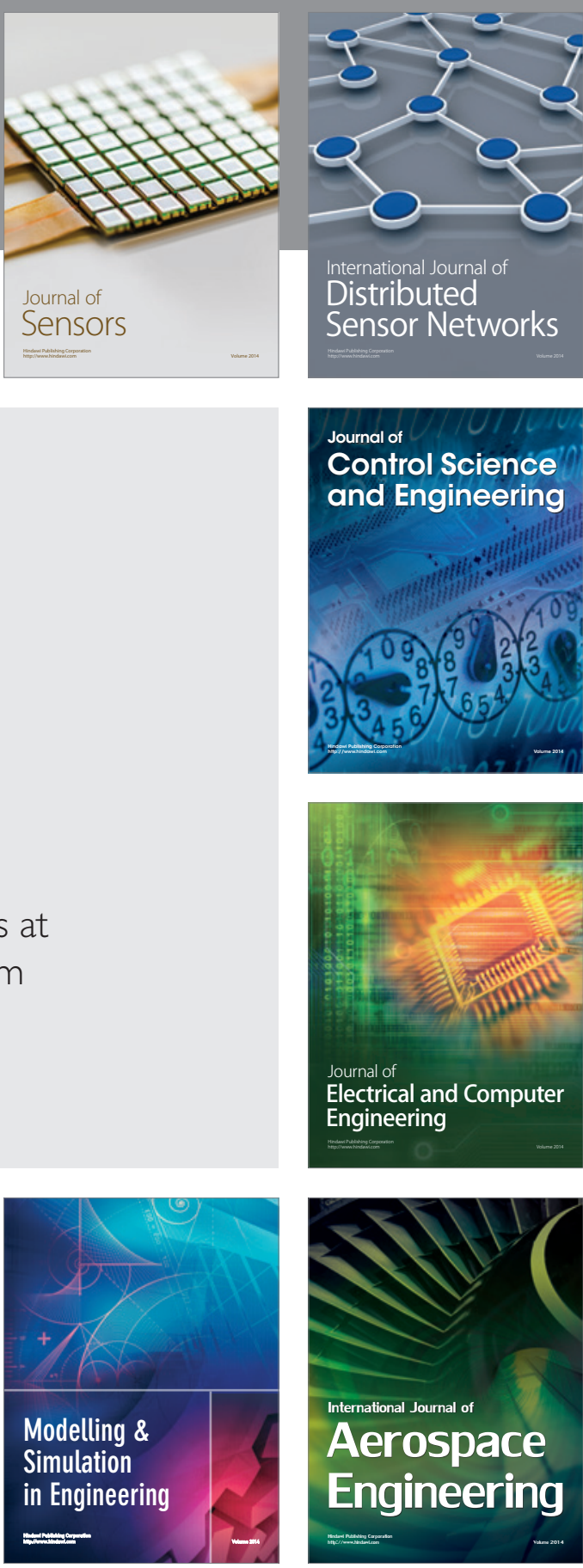

International Journal of

Distributed

Sensor Networks

Journal of

Control Science

and Engineering
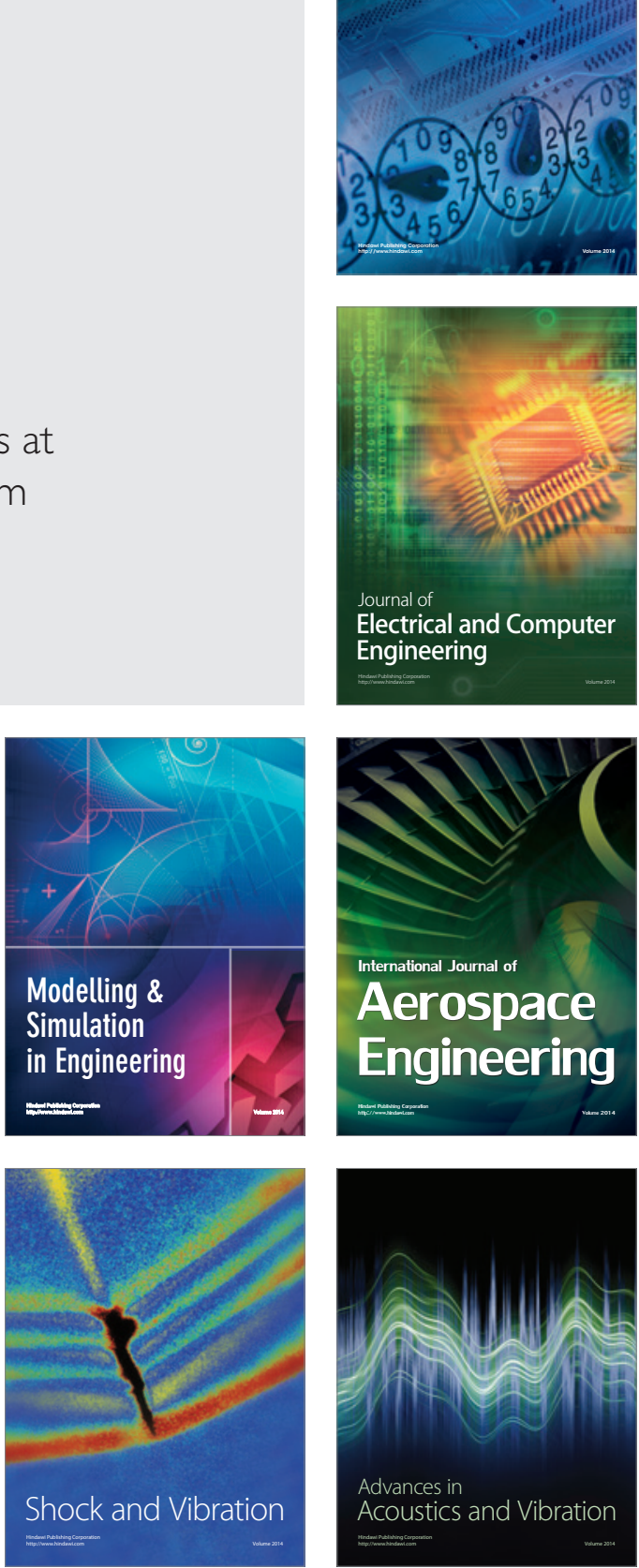